\title{
Relation between Left Atrial Remodeling in Young Patients with Cryptogenic Stroke and Normal Inter-atrial Anatomy
}

\author{
Mustafa Gökhan Vural, ${ }^{a}$ Suha Cetin, ${ }^{\mathrm{b}}$ Murat Yilmaz, ${ }^{\mathrm{c}}$ Ramazan Akdemir, ${ }^{\mathrm{a}}$ Huseyin Gunduz ${ }^{\mathrm{a}}$ \\ a Sakarya University School of Medicine, Cardiology Department, Sakarya, Turkey \\ 'Koru Private Hospital, Cardiology Department, Ankara, Turkey \\ 'Ankara Numune Research and Education Hospital, Neurology Department, Ankara, Turkey
}

Background and Purpose To investigate an association between left atrial (LA) structural and $\mathrm{P}$ wave dispersion (PWD) during sinus rhythm, and electrical remodeling in cryptogenic stroke (CS) patients.

Methods Forty CS patients and 40 age- and sex-matched healthy controls were enrolled. P wave calculations were based on 12-lead electrocardiography (ECG) at a $50-\mathrm{mm} / \mathrm{s}$-paper speed with an amplitude of $10 \mathrm{~mm} / \mathrm{mV}$. Difference between the maximum and minimum $P$ wave duration was the $\mathrm{P}$ wave dispersion (PWD = Pmax-Pmin). LA deformation was evaluated by speckle tracking echocardiography within 3 days of the acute event.

Results PWD was $30.1 \pm 7.0 \mathrm{~ms}$ and $27.4 \pm 3.5 \mathrm{~ms}$ in CS and control group $(P=0.02)$, whereas LA maximum volume index [LAVImax] was $20.4 \pm 4.5 \mathrm{~mL} / \mathrm{m}^{2}$ and $19.9 \pm 2.4 \mathrm{~mL} / \mathrm{m}^{2}$ in CS and control group, respectively $(P=0.04)$. While global peak LA strain was [pLA-S] (LA reservoir function) $41.4 \pm 6.3 \%$ and $44.5 \pm 7.1 \%$ in $\mathrm{CS}$ and control group, $(P=0.04)$, global peak late diastolic strain rate values [pLA-SRa] (LA pump function) were $2.5 \pm 0.4 \%$ and $2.9 \pm 0.5 \%$ in $\mathrm{CS}$ and control group, respectively $(P=0.001)$. A mild and a strong negative correlation between global pLA-S and LAVImax $(r=-0.49 ; P<0.01)$, and between PWD and global pLA-S $(r=-0.52 ; P<0.01)$, respectively, was observed in CS.

Conclusions Increased PWD is associated with impaired LA mechanical functions and enlargement, and involved in the pathophysiology of AF or an AF-like physiology in CS.

Keywords Cryptogenic stroke; P wave dispersion; Echocardiography; Paroxysmal atrial fibrillation; Strain

\author{
Correspondence: Mustafa Gokhan Vural \\ Sakarya Universitesi Tip Fakultesi \\ Dekanligi, Korucuk Mahallesi Konuralp \\ Bulvari No: 81/1 Turkey \\ Tel: +90-(264)-295-66-30 \\ Fax: +90-(264)-295-66-29 \\ E-mail:mgvural@sakarya.edu.tr
}

Received: November 16, 2014

Revised: January 19, 2015

Accepted: January 26, 2015

The authors have no financial conflicts of interest.

\section{Introduction}

Cryptogenic stroke (CS) refers to a cerebral ischemia of unknown origin. ${ }^{1}$ While atrial fibrillation (AF) is the most common associated cardiac arrhythmia making up to $12 \%$ of strokes among the general population, ${ }^{2}$ asymptomatic paroxysmal AF
(PAF) is often suspected as the underlying cause of CS. ${ }^{3}$ The progressive nature of $\mathrm{AF}$ is well established and known to lead to electro-anatomical remodeling and atrial fibrosis. ${ }^{4}$ Atrial fibrosis develops from complex interactions among several cellular and neurohumoral mediators, with the resultant fibrotic tissue causing a low a voltage response and other electrophysio- 
logical changes in the left atrium (LA) that act as a substrate for the occurrence of AF. While electrical remodeling may shorten atrial refractoriness and contributes to an increase in the stability of AF, atrial structural remodeling occurs because of heart failure and other underlying cardiovascular diseases. Moreover, LA fibrosis has been associated with an increased risk of developing a stroke in the absence of AF or PAF, as shown by magnetic resonance imaging (MRI). ${ }^{5}$

Speckle tracking echocardiography, a new imaging technique that offers offline calculation of myocardial velocities and deformation parameters such as strain and strain rate (SR), provides important insights into systolic and diastolic function, ischemia, myocardial mechanics, and many other pathophysiological heart processes..$^{6.9}$ A sensitive, noninvasive, reproducible, and quantitative assessment of LA longitudinal deformation, which is closely related to LA physiology and anatomy, is achieved by means of this technique. ${ }^{6-9}$

LA longitudinal deformation indices (global peak LA strain [pLA-S], global peak LA early diastolic strain rate [pLA-SRe], and global peak LA late diastolic strain rate [pLA-SRa]) appear to provide the most accurate physiologic and anatomic analysis of LA.

As decreased LA longitudinal deformation has been closely associated with PAF, its detection allows the identification of patients with a high risk of $\mathrm{AF}$ recurrence after catheter ablation procedures, irrespective of LA enlargement. ${ }^{9,10}$ Furthermore, decreased LA longitudinal deformation predicts the extent of LA fibrosis independent of other echocardiographic parameters, including rhythm. ${ }^{11,12}$ Therefore, characterization of LA fibrosis using speckle-tracking echocardiography may aid in the diagnosis of CS.

Recently, an association between a decreased LA ejection fraction (LA EF) and an increased risk of PAF in CS has been suggested. ${ }^{13}$ Furthermore, LA active relaxation and contraction is lower in PAF patients than in those with sinus rhythm, regardless of LA enlargement and aging.

$\mathrm{P}$ wave dispersion (PWD), defined as the difference between the maximum (Pmax) and the minimum (Pmin) P wave duration on electrocardiography (ECG), has been regarded as an independent risk factor for the development of $\mathrm{AF}^{14,15}$ As PWD may be easily measured using a single ECG, it is considered an electrocardiographic marker of atrial electromechanical dyssynchrony caused by the heterogeneous propagation of sinus impulses. On the other hand, PWD determined within the first day after of an acute ischemic stroke serves as a surrogate marker to predict PAF and the risk of recurrent strokes. ${ }^{16}$

The aim of this study was to investigate associations between LA electro-structural remodeling and the occurrence of CS.

\section{Methods}

\section{Patients and data collection}

In this cross-sectional study, we have used speckle tracking echocardiography and ECG to assess LA structural and electrical remodeling, and examined its association with the occurrence of CS.

All consecutive patients presenting with a stroke of unknown origin despite extensive routine diagnostic testing and diagnosed with CS between 2009 and 2010, were included in the study. Strokes were classified according to the TOAST criteria. ${ }^{17}$

We excluded patients who had a previous cerebrovascular event, a TOAST Classification of High- and Medium-Risk Sources of Cardioembolism, or malignant arterial hypertension; uncontrolled diabetes mellitus; a history of effort angina, acute coronary syndrome, or revascularization procedures; evidence of a positive exercise stress test before the acute stroke; segmental wall abnormalities at echocardiography; presence or 24-hourholter detected $\mathrm{AF}$, atrial flutter, or other major arrhythmias; moderate-to-severe valvulopathies, existence of inter-atrial block on 12-lead ECG, or inter-atrial septum abnormalities; patients who had a malign clinical course in the neurology intensive care unit, including severe kidney, respiratory, cardiac or hepatic failure, and inadequate acoustic windows were excluded. Patients who required intubation because of respiratory insufficiency, and those who required more than 3 days in a neurology intensive care unit were also excluded.

All patients fulfilled both the inclusion and exclusion criteria. From those, 40 (aged between 18 and 55 years) who were admitted to our emergency department and hospitalized in the neurology intensive care unit with a diagnosis of acute ischemic stroke, and a control group comprised of 40 age- and sex-matched healthy individuals, were enrolled in the study. Participants in the control group underwent a complete routine clinical and cardiac laboratory evaluation for the detection of occult cardiac disease, including 12-lead ECG, transthoracic/transesophageal echocardiography (TTE/TEE), extra cranial arteries duplex sonography, and single ECG-24-hour Holter ECG monitoring before inclusion in the study. Individuals with any cardiac structural pathology and arrhythmia, including patent foramen ovale, atrial septal aneurysm, or paroxysmal atrial fibrillation, were excluded from the study.

The following routine diagnostic tests were performed in all patients: cranial computed tomography and magnetic resonance imaging of the brain, or both. Duplex sonography of the extra cranial and intracranial arteries, a single ECG-24-hour Holter ECG monitoring, and TTE/TEE were performed in patients within a median of 3 days after the acute stroke. 


\section{LA structural remodeling analysis using conventional and speckle tracking echocardiography}

Electrocardiogram-guided echocardiographic measurements were carried out using a commercially available ultrasound device (ie33, Philips Medical System, Bothell, Washington, USA). Images were obtained from parasternal and apical windows using 2D, M-Mode, and Doppler echocardiography.

The LVEF, as a standard index of global LV systolic function, was measured using the Simpson's method. The ratio between the peaks of early (E) and late (A) diastolic LV filling velocities was used as the standard index of LV diastolic function. ${ }^{18}$ Tissue Doppler measurements were obtained at end-expiration with the sample volume placed on the atrial side of the mitral annulus at the basal inter-atrial septum in the apical four-chamber view. Both early diastolic $\left(\mathrm{E}^{\prime}\right)$, and late diastolic $\left(\mathrm{A}^{\prime}\right)$ annular velocities were obtained. The $\mathrm{E} / \mathrm{E}^{\prime}$ ratio was also calculated to provide a reliable index of the LV filling pressure.

At end-systole, just before the opening of the mitral valve (at the end of the T wave on the ECG), the LA maximum volume was indexed to body surface area (LAVImax). At end-diastole, just before mitral valve closure (at the beginning of the QRS complex on the ECG), minimum LA volume to body surface area (LAVImin) were measured and indexed to body surface area, as previously described. ${ }^{19}$

For speckle tracking echocardiography analysis, apical 4- and 2-chamber view images were obtained using conventional 2-dimensional gray-scale echocardiography during breath-hold. Care was taken to obtain a reliable delineation of the atrial endocardial border. The frame rate was set at > 100 frames/s. Three consecutive heart cycles were recorded in digital format for offline analysis using the commercially available software QLAB 6.0 (Philips Medical System, Bothell, Washington, USA). Segments for which adequate tracking quality could not be obtained despite manual adjustment, were excluded from analysis, and patients in whom some segments were excluded due to the impossibility of adequate tracking, LA deformation parameters were calculated by averaging the values measured in the remaining segments. LA peak longitudinal strain and strain rate at basal segment from the apical views of the inter-atrial septum, LA lateral wall, LA anterior wall, and LA inferior wall in the $5 \times 3 \mathrm{~mm}$ region of interest were calculated as described in current guidelines. ${ }^{8}$ LA reservoir strain during systole was obtained at the time of aortic valve closure, and LA contractile strain rate during late diastole was obtained at the onset of the P wave on ECG. LA conduit strain rate during early diastole was obtained as previously described. The reproducibility and feasibility of speckle tracking echocardiography measurement of LA longitudinal strain and strain rates were considered acceptable.

\section{LA electrical remodeling analysis using $\mathrm{P}$ wave dispersion}

All standard 12-lead ECGs were obtained simultaneously at a $50-\mathrm{mm} / \mathrm{s}$-paper speed and amplitude of $10 \mathrm{~mm} / \mathrm{mV}$. Two cardiologists blind to the clinical status of the patients performed the $\mathrm{P}$ wave analysis with calipers and magnifying glasses to decrease the risk of measurement errors. The onset of the $\mathrm{P}$ wave was defined as the point in which the wave showed the first visible upward and downward departure from baseline for positive and negative waveforms, respectively. A return to the baseline was considered the end of the $\mathrm{P}$ wave. Maximum $\mathrm{P}$ wave duration (Pmax), measured from any of the 12 leads of the surface ECG, was used for the longest atrial conduction time. The difference between the maximum and minimum $\mathrm{P}$ wave duration was defined as PWD (PWD= Pmax-Pmin). Intraobserver and interobserver coefficients of variation were acceptable. Written informed consent was obtained from each patient. The local ethics committee approved the study.

\section{Statistical analysis}

Data have been presented as mean \pm SD for continuous variables and as proportions for categorical variables. For continuous data, statistical differences were evaluated using a Student's $t$ test, or alternatively a Mann-Whitney $U$ test for cases in which the assumptions of the Student's $t$ test were not satisfied. For categorical data, the chi-square test was used. All correlations were analyzed using a Spearman's rank correlation test. A $P$ value $<0.05$ was considered statistically significant. All statistical analyses were performed using SPSS statistical software, version 15.0 (SPSS Inc., Chicago, IL, USA).

\section{Results}

Clinical and conventional echocardiographic characteristics of the study population are shown in Table 1. Patients with high blood pressure and high glucose levels received appropriate medication. No significant differences with regard to age, gender, body mass index, body surface area, heart rate, blood pressure, left atrial minimal volume index, and left ventricular ejection fraction, were observed between the two groups. The PWD was $30.1 \pm 7.0 \mathrm{~ms}$ and $27.4 \pm 3.5 \mathrm{~ms}$ in the CS and control group, respectively $(P=0.02)$, whereas the LA maximum volume index [LAVImax] was $20.4 \pm 4.5 \mathrm{~mL} / \mathrm{m}^{2}$ and $19.9 \pm 2.4 \mathrm{~mL} / \mathrm{m}^{2}$ in the $\mathrm{CS}$ and control group, respectively $(P=0.04)$. The global peak LA strain was [pLA-S] (LA reservoir function) $41.4 \pm 6.3 \%$ and $44.5 \pm 7.1 \%$ in the CS and control group, respectively $(P=0.04)$, whereas the global peak late diastolic strain rate values [pLASRa] (LA pump function) were $2.5 \pm 0.4 \%$ and $2.9 \pm 0.5 \%$ in the 
$\mathrm{CS}$ and control group, respectively $(P=0.001)$. A strong negative correlation between global pLA-S and LAVImax $(r=-0.49$; $P<0.01)$, as well as a moderate correlation between PWD and global pLA-S $(r=-0.52 ; P<0.01)$, was found in CS patients (Figures 1,2$)$. Although there was no significant difference in left atrial tissue Doppler velocities between patients and healthy controls, global pLA-S was found to be significantly lower in CS patients (Table 2). A significant difference was found in the pattern of LA segment changes in CS patients (Table 2 and Figure 3).

Table 1. General characteristics of the study population

\begin{tabular}{|c|c|c|c|}
\hline Variables & $\begin{array}{l}\text { Control group } \\
\qquad(n=40)\end{array}$ & $\begin{array}{l}\text { Cryptogenic stroke pa- } \\
\text { tients }(n=40)\end{array}$ & $P$ value \\
\hline Age (year) & $42.5 \pm 7.1$ & $41.9 \pm 6.7$ & 0.702 \\
\hline $\operatorname{Sex}(m / f)$ & $20 / 20$ & $20 / 20$ & 1.000 \\
\hline $\mathrm{BSA}\left(\mathrm{m}^{2}\right)$ & $1.8 \pm 0.2$ & $1.8 \pm 0.2$ & 0.920 \\
\hline $\mathrm{BMI}\left(\mathrm{kg} / \mathrm{m}^{2}\right)$ & $26.2 \pm 3.0$ & $26.1 \pm 2.9$ & 0.869 \\
\hline Hypertension (\%) & $14(35)$ & $17(42)$ & 0.491 \\
\hline Diabetes mellitus (\%) & $4(10)$ & $3(7)$ & 0.692 \\
\hline Smoking (\%) & $17(42)$ & $14(35)$ & 0.491 \\
\hline Hypercholesterolemia (\%) & $10(25)$ & $13(32)$ & 0.459 \\
\hline $\begin{array}{l}\text { Systolic blood pressure } \\
(\mathrm{mmHg})\end{array}$ & $120.7 \pm 10.7$ & $120.5 \pm 11.3$ & 0.919 \\
\hline $\begin{array}{l}\text { Diastolic blood pressure } \\
(\mathrm{mmHg})\end{array}$ & $73.2 \pm 8.8$ & $71.0 \pm 11.0$ & 0.319 \\
\hline $\operatorname{LVEF}(\%)$ & $64.5 \pm 2.7$ & $65.6 \pm 2.9$ & 0.075 \\
\hline $\begin{array}{l}\text { LA maximal volume index } \\
\left(\mathrm{mL} / \mathrm{m}^{2}\right)\end{array}$ & $19.9 \pm 2.4$ & $20.4 \pm 4.5$ & 0.047 \\
\hline $\begin{array}{l}\text { LA minimal volume index } \\
\left(\mathrm{mL} / \mathrm{m}^{2}\right)\end{array}$ & $10.1 \pm 2.6$ & $10.5 \pm 2.8$ & 0.254 \\
\hline $\begin{array}{l}\text { Myocardial diastolic early } \\
\text { velocity }\left(\mathrm{E}^{\prime}\right)\end{array}$ & $8.7 \pm 2.9$ & $8.0 \pm 3.0$ & 0.095 \\
\hline $\begin{array}{l}\text { Myocardial diastolic late } \\
\text { velocity }\left(A^{\prime}\right)\end{array}$ & $7.6 \pm 2.8$ & $7.1 \pm 2.3$ & 0.163 \\
\hline$P \max$ & $89.5+8.8(80.5-98.8)$ & $98.3+6.4(91.6-105.7)$ & 0.006 \\
\hline$P \min$ & $56.1+6.7(50.3-62.2)$ & $58.0+8.3(51.2-64.6)$ & 0.054 \\
\hline PWD & $27.4 \pm 3.5(24.2-31.6)$ & $30.1 \pm 7.0(23.3-40.4)$ & 0.029 \\
\hline
\end{tabular}

$M$, male; f, female; BSA, body surface area; BMl, body mass index; LV, left ventricle; EF, ejection fraction; LA, left atrium; PWD, P wave dispersion.

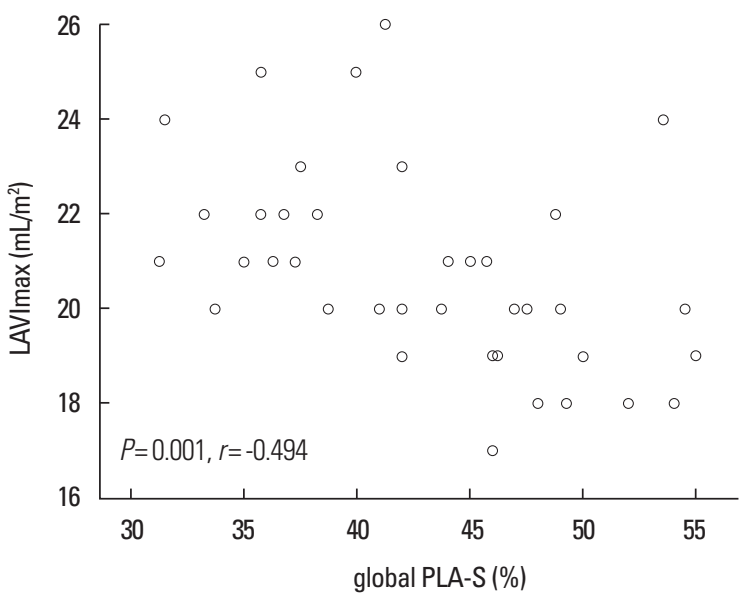

Figure 1. A strong negative correlation between global pLA-S and LAVImax $(r=-0.49 ; P<0.01)$ was found in CS patients.
The LA reservoir function (LA strain) was smaller in CS patients than in healthy controls. The inter-atrial septal and inferior wall early diastolic strain rates (LA conduit function) and the anterior late diastolic strain rate (LA pump function) were significantly lower in CS patients than in healthy controls, whereas the interatrial septal late diastolic strain rate tended to be lower but the difference was not statistically significant.

\section{Discussion}

In this study we have demonstrated that electrical remodeling, shown by an increase in PWD on surface ECG, was associated with structural remodeling and decreased LA deformation shown on echocardiography. These findings suggest that LA

Table 2. Myocardial deformation properties in cryptogenic stroke patients compared with healthy subjects

\begin{tabular}{lccc}
\hline Variables & $\begin{array}{c}\text { Control group } \\
(\mathrm{n}=40)\end{array}$ & $\begin{array}{c}\text { Cryptogenic stroke } \\
\text { patients }(\mathrm{n}=40)\end{array}$ & Pvalue \\
\hline $\begin{array}{l}\text { Left atrial strain }(\%) \\
\text { Inter-atrial septum }\end{array}$ & $59.1 \pm 13.0$ & $53.0 \pm 12.1$ & 0.034 \\
Lateral wall & $34.9 \pm 7.9$ & $32.6 \pm 8.2$ & 0.193 \\
Anterior wall & $43.2 \pm 9.9$ & $38.9 \pm 7.8$ & 0.037 \\
$\quad$ Inferior wall & $43.4 \pm 9.0$ & $39.0 \pm 8.4$ & 0.027 \\
Global & $44.5 \pm 7.1$ & $41.4 \pm 6.3$ & 0.043 \\
Left atrial strain rate, e $\left(\mathrm{s}^{-1}\right)$ & & & \\
Inter-atrial septum & $3.1 \pm 0.6$ & $2.9 \pm 0.6$ & 0.042 \\
Lateral wall & $3.0 \pm 0.6$ & $2.8 \pm 0.5$ & 0.219 \\
Anterior wall & $3.2 \pm 0.7$ & $2.9 \pm 0.7$ & 0.060 \\
Inferior wall & $3.2 \pm 0.7$ & $2.8 \pm 0.8$ & 0.030 \\
Global & $3.1 \pm 0.4$ & $2.9 \pm 0.4$ & 0.056 \\
Left atrial strain rate, a $\left(\mathrm{s}^{-1}\right)$ & & & \\
Inter-atrial septum & $3.0 \pm 1.1$ & $2.6 \pm 0.8$ & 0.048 \\
Lateral wall & $2.9 \pm 0.8$ & $2.6 \pm 0.7$ & 0.065 \\
Anterior wall & $3.0 \pm 0.9$ & $2.6 \pm 0.8$ & 0.034 \\
Inferior wall & $2.6 \pm 0.5$ & $2.4 \pm 0.5$ & 0.119 \\
Global & $2.9 \pm 0.5$ & $2.5 \pm 0.4$ & 0.001 \\
\hline
\end{tabular}

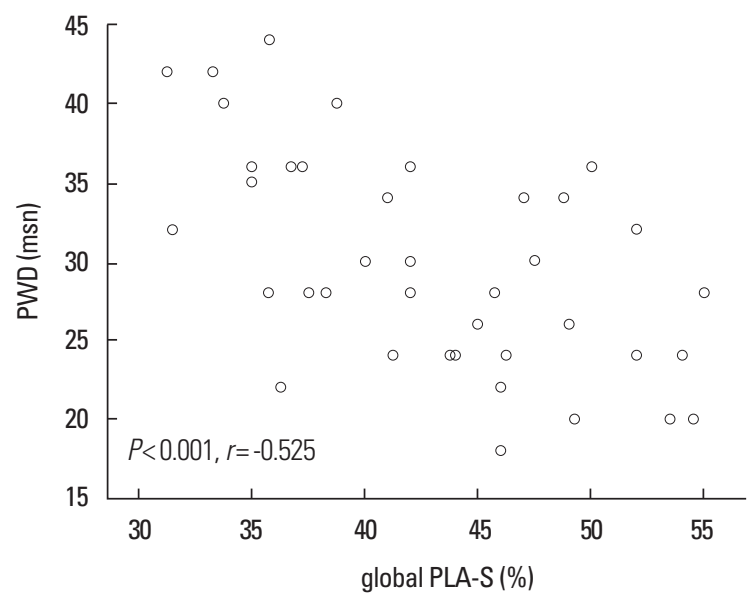

Figure 2. A moderate correlation between PWD and global pLA-S ( $r=-0.52$; $P<0.01$ ) was found in CS patients. 


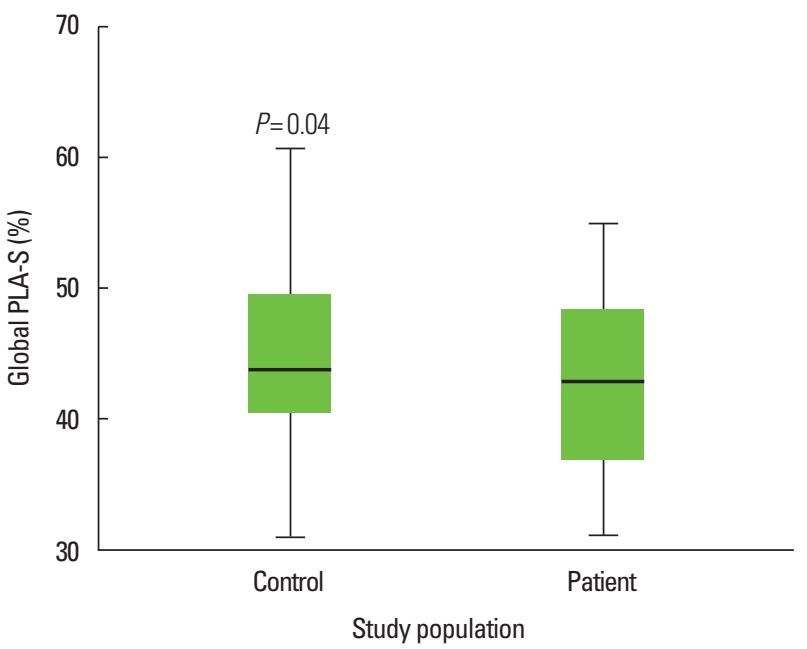

Figure 3. The global peak LA strain values in cryptogenic stroke patients compared with healthy subjects.

structural, functional, and electrical impairments in conjunction with other risk factors i.e. increased thrombogenicity and a heightened proinflammatory response, may ultimately result in cardioembolism in patients without inter-atrial septal abnormalities. PWD was increased in CS patients mainly due to an increase in maximum $P$ wave values rather than a decrease in minimum $P$ wave durations on a 12-lead ECG, and it was associated with an increased risk of AF development. Although, the increase in PWD and structural changes observed in LA may explain the occurrence of CS, whether these changes are an underlying cause or an associated finding in the early post-stroke period (within 1 week) in CS patients, remains unclear. ${ }^{18-20}$

Furthermore, AF results in LA remodeling through a process that involves the deposition of fibrotic tissue, leading to changes in the electrophysiological properties of the LA substrate. Detection and quantification of LA structural remodeling as a possible determinant of fibrosis, is now possible using delayed-enhancement magnetic resonance imaging. Nevertheless, whether substrate changes seen in AF patients are related to stroke and current stroke risk stratification schemes is not yet clear.

The prolongation of intra- and inter-atrial conduction times and the inhomogeneous propagation of sinus impulses are well known electrophysiological characteristics seen in patients with PAF. ${ }^{15,16}$ PWD has proven to be a sensitive and specific ECG predictor of AF in various clinical settings, including patients with hypertension, coronary artery disease, undergoing coronary artery bypass surgery, or obstructive sleep apnea. ${ }^{20-23} \mathrm{In}$ creases in PWD and Pmax are important indicators of subsequent PAF attacks and a tendency towards persistent atrial fibrillation. Furthermore, an association between incremental LA enlargement and an increased risk of AF during follow-up has been suggested, with the risk of developing AF being four times greater in patients with enlarged LA.,5 Results from studies by Abhayaratna et al. ${ }^{24}$ showed that increased LAVImax was an independent predictor of death, heart failure, atrial fibrillation, and ischemic stroke.

The role of LA function in stroke pathogenesis has been supported by recent data showing that inter-atrial septal abnormalities lead to LA dysfunction, which may in turn contribute to LA thrombosis and subsequent ischemic stroke. ${ }^{25,26}$ Moreover, Goch et al. ${ }^{27}$ showed that patients with only atrial septum aneurysm (ASA) had depressed LA systolic function and increased left atrial appendage function, which might be a compensatory mechanism for LA deterioration. Jin et al. revealed that LA active pump function was significantly depressed, and closely correlated with left atrial appendage emptying in CS patients with ASA alone. ${ }^{3}$ Therefore, impaired LA and LA appendage (LAA) functions may be crucial pathophysiologic mechanisms for ischemic stroke in patients with ASA alone.

To our knowledge, LA structure and function in CS patients without inter-atrial septal abnormalities have not been thoroughly investigated. The present study provides new insights into LA remodeling in patients with CS, demonstrating that global pLA-S, a newer parameter of atrial function, is more sensitive than the traditional echocardiographic markers of LA size and function used to detect LA remodeling.

Routine measurement of LA strain might guarantee the detection of LA remodeling, and provide useful additional information for CS diagnosis. Thus, this novel imaging method may be considered a promising index for a more accurate quantification of LA function, allowing the potential identification of LA impairment, which may be a useful parameter for CS risk stratification. However, as this novel technique has insufficient resolution to measure the radial strain of the thin-walled LA, LA deformation assessment is only based on longitudinal strain. Longitudinal strain is positive during the reservoir period, in which LA myocardial fibers relax and stretch to adapt to the incoming blood flow, negative during the pump and most of the conduit phase, during which the LA is emptying, and flat during diastasis, corresponding to the late phase of the conduit phase. Therefore, measurements taken at end-systole, as well as early and late end-diastole, may provide information about the reservoir, conduit, and pump functions. Furthermore, global pLA-S has considerably high diagnostic accuracy while the $\mathrm{E} / \mathrm{E}^{\prime}$ ratio correlates poorly with invasively measured LA pressure. In patients with $\mathrm{AF}$ who underwent catheter ablation, the systolic, as well as the early and late diastolic strains, were considerably lower in patients with persistent $\mathrm{AF}$ than in patients with PAF or normal subjects. ${ }^{10}$ Similarly, lower strain and strain rate were associated with a higher rate of AF reoccurrence after catheter ablation, 
and an improvement in LA strain correlated with a reversion of LA remodeling in patients who underwent catheter ablation for AF. This latter finding may have essential prognostic and therapeutic implications concerning the post procedure cardioembolic risk and oral anticoagulation therapy.

Despite the obvious advantages of two-dimensional echocardiography, the difficulty of endocardial border tracing and its reliance on geometrical assumptions, which ignore LV and LA geometrical differences between individuals, limits its application. However, LA strain measurements may facilitate atrial remodeling assessment in various pathological conditions, as well as reverse remodeling after medical or more invasive therapy, such as cardiac resynchronization therapy, ablation for atrial fibrillation or flutter, hypertrophic cardiomyopathy, or coronary artery diseases. ${ }^{28-31}$ In support of this view, Karabay et al. ${ }^{32}$ recently demonstrated that LA deformation parameters measured by speckle tracking were found to predict impaired LA appendage (LAA) functions and the presence of LAA thrombus in ischemic stroke patients with suspected cardioembolism in sinus rhythm. In that study, LA reservoir and pump functions were found to be significantly lower in patients with LAA thrombus, and the LA reservoir function showed the strongest correlation with LAA morphologic parameters.

Pagola et al. ${ }^{33}$ recently claimed that measurement of pLA-S in patients with CS might be a useful tool to detect patients with occult PAF. Furthermore, they concluded that PLA-S analysis may play a role in the selection of patients with normal size LA for prolonged cardiac monitorization, because most patients with PAF showed low LAS in their study. Although, their results were similar, the patients mean age of the study population was 62 years, whereas in our study it was only 42 years. Since aging is associated with atrial fibrosis and atrial arrhythmias, ${ }^{34}$ we have excluded an aging effect on left atrial anatomy and function in CS patients in this study. For the first time, we have offered LA remodeling in young CS patients.

LA structural remodeling, as demonstrated by the histopathology findings, significantly influences global pLA-S. ${ }^{35}$ Therefore, assessment of LA function and LA ultrastructural changes may provide additional information for the prediction of cardiovascular events. In particular, atrial fibrosis has been strongly associated with the presence of heart disease and arrhythmias, including congestive heart failure and atrial fibrillation. Moreover, an adverse prognosis linked to progressive LA substrate remodeling, showed a correlation between stroke and high levels of LA fibrosis, detected by delayed enhancement MRI in patients with AF. However, global pLA-S measured by speckle tracking echocardiography was negatively correlated with LA myocardial fibrosis grade, while poorer correlations with the
LA indexed volume, LA ejection fraction, and $\mathrm{E} / \mathrm{E}^{\prime}$ ratio have been shown.

As global pLA-S is also correlated with endocardial thickening, a histologic alteration appearing in the earlier stages of structural remodeling, ${ }^{35}$ it can be used to assess an increase in interstitial fibrosis in conditions which compromise the elastic properties of the atrial myocardium, inevitably leading to impairment of atrial compliance and reduction of LA reservoir function.

Although our findings are in general agreement with the results of previous studies, there are several notable differences. Compared with our study, most studies selected subjects from the general population who presented inter-atrial septal abnormalities. Therefore, their results are not representative of all the underlying pathophysiologic mechanisms operating in the development of ischemic events in CS patients with no inter-atrial septal abnormalities. Furthermore, in order to eliminate possible etiologies associated with paradoxical embolism and to prevent misleading results, we only included CS patients without PFO or ASA on TEE.

\section{Limitations}

The main limitation of our study the inability to demonstrate a clear relationship between LA structure and LA strain using histopathological or delayed enhancement MRI.

Secondly, to exclude undetected paroxysmal AF (PAF), in addition to a single 24-hour Holter monitoring in all subjects, we performed standard 12-lead ECGs at every visit as well as at any time if the subjects reported palpitations. However, we were not able to exclude PAF episodes reliably because they are often asymptomatic, and those undetected episodes may have contributed to confounding during the statistical analysis of LA function.

Although the effect of various drugs, including anti-thrombotic drugs, angiotensin converting enzyme inhibitors or angiotensin receptor blockers, and statins, on LA function or plasma biomarkers could not be fully controlled, as not all subjects were receiving the same drug therapies, there were no significant differences in medication regimens between the two study groups.

Some cardiac abnormalities that cannot be detected by routine diagnostic methods may be a cause of AF or PAF and subsequent CS. ${ }^{36}$

Acute cerebral events such as subarachnoid hemorrhage may cause stunned myocardium, a neurogenic cardiac dysfunction, as well as arrhythmia and ischemia changes during the acute phase. Although the mechanisms responsible for those changes are unknown, the release of stress hormones, adrenaline, and nor-adrenaline, caused by major stressful events, is suspected. ${ }^{37,38}$

Lastly, we cannot completely exclude the existence of right-to- 
left shunt, as exclusion of PFO was only confirmed by TEE using contrast bubbles. Recently, transcranial Doppler examination has been considered more sensitive than TEE for the diagnosis of a right-to-left shunt. Nevertheless, our hypothesis that LA dysfunction itself may contribute to thrombosis in situ through an embolism with an extra-cardiac origin remains plausible. Therefore, further interventional studies are warranted to investigate whether improvement of LA function by reversion of LA remodeling would protect from ischemic stroke in the general population.

\section{Conclusions}

Increased PWD on surface ECG is associated with left atrial mechanical remodeling and dysfunction, both of which may be substrates for thromboembolism in CS patients.

\section{References}

1. Vallejos J, Jaramillo A, Reyes A, Illanes S, Orellana P, Manterola J, et al. Prognosis of cryptogenic ischemic stroke: a prospective single-center study in Chile. J Stroke Cerebrovasc Dis 2012;21: 621-628.

2. Kishore A, Vail A, Majid A, Dawson J, Lees KR, Tyrrell PJ, et al. Detection of atrial fibrillation after ischemic stroke or transient ischemic attack: a systematic review and meta-analysis. Stroke 2014;45:520-526.

3. Flint AC, Banki NM, Ren X, Rao VA, Go AS. Detection of paroxysmal atrial fibrillation by 30 -day event monitoring in cryptogenic ischemic stroke: the Stroke and Monitoring for PAF in Real Time (SMART) Registry. Stroke 2012;43:27882790.

4. Marrouche NF, Wilber D, Hindricks G, Jais P, Akoum N, Marchlinski F, et al. Association of atrial tissue fibrosis identified by delayed enhancement MRI and atrial fibrillation catheter ablation: the DECAAF study. JAMA 2014;311:498-506.

5. Russo C, Jin Z, Liu R, Iwata S, Tugcu A, Yoshita M, et al. LA volumes and reservoir function are associated with subclinical cerebrovascular disease: the CABL (Cardiovascular Abnormalities and Brain Lesions) study. JACC Cardiovasc Imaging 2013; 6:313-323.

6. Vianna-Pinton R, Moreno CA, Baxter CM, Lee KS, Tsang TS, Appleton CP. Two-dimensional speckle-tracking echocardiography of the left atrium: feasibility and regional contraction and relaxation differences in normal subjects. J Am Soc Echocardiogr 2009;22:299-305.

7. Kucuk U, Kucuk HO, Demirkol S, İyisoy A. Left atrial deformation parameters predict left atrial appendage function and thrombus in patients in sinus rhythm with suspected cardioembolic stroke: a speckle tracking and transesophageal echocardiography study: left atrial filling and emptying velocities as indicators of left atrial mechanics. Echocardiography 2013;30: 860-861.

8. Vural MG, Cetin S, Firat H, Akdemir R, Yeter E. Impact of continuous positive airway pressure therapy on left atrial function in patients with obstructive sleep apnea: assessment by conventional and two-dimensional speckle-tracking echocardiography. Acta Cardiol 2014;69:175-184.

9. Tsai WC, Lee CH, Lin CC, Liu YW, Huang YY, Li WT, et al. Association of left atrial strain and strain rate assessed by speckle tracking echocardiography with paroxysmal atrial fibrillation. Echocardiography 2009;26:1188-1194.

10. Motoki H, Negishi K, Kusunose K, Popović ZB, Bhargava M, Wazni OM et al. Global left atrial strain in the prediction of sinus rhythm maintenance after catheter ablation for atrial fibrillation. J Am Soc Echocardiogr 2014;27:1184-1192.

11. Cameli M, Lisi M, Righini FM, Massoni A, Natali BM, Focardi $\mathrm{M}$, et al. Usefulness of atrial deformation analysis to predict left atrial fibrosis and endocardial thickness in patients undergoing mitral valve operations for severe mitral regurgitation secondary to mitral valve prolapse. Am J Cardiol 2013;111:595-601.

12. Her AY, Choi EY, Shim CY, Song BW, Lee S, Ha JW, et al. Prediction of left atrial fibrosis with speckle tracking echocardiography in mitral valve disease: a comparative study with histopathology. Korean Circ J 2012;42:311-318.

13. Na JO, Shin SY, Lim HE, Choi CU, Kim SH, Kim JW, et al. Impaired transport function of the left atrium and left atrial appendage in cryptogenic stroke patients with atrial septal aneurysm and without patent foramen ovale. Eur J Echocardiogr 2011;12:140-147.

14. Akutsu Y, Kaneko K, Kodama Y, Miyoshi F, Li HL, Watanabe $\mathrm{N}$, et al. A combination of $\mathrm{P}$ wave electrocardiography and plasma brain natriuretic peptide level for predicting the progression to persistent atrial fibrillation: comparisons of sympathetic activity and left atrial size. J Interv Card Electrophysiol 2013;38: 79-84.

15. Villani GQ, Piepoli M, Rosi A, Capucci A. P-wave dispersion index: a marker of patients with paroxysmal atrial fibrillation. Int J Cardiol 1996;55:169-175.

16. Dogan U, Dogan EA, Tekinalp M, Tokgoz OS, Aribas A, Akilli $\mathrm{H}$, et al. P-wave dispersion for predicting paroxysmal atrial fibrillation in acute ischemic stroke. Int J Med Sci 2012;9:108-114.

17. Adams HP Jr, Bendixen BH, Kappelle LJ, Biller J, Love BB, Gordon DL, et al. Classification of subtype of acute ischemic stroke. Definitions for use in a multicenter clinical trial. TOAST. Trial of Org 10172 in Acute Stroke Treatment. Stroke 1993;24: 
35-41.

18. Nahun LH. Neurogenic effects on the electrocardiographic wave forms. Conn Med 1971;35:556-558.

19. Oppenheimer SM. Neurogenic cardiac effects of cerebrovascular disease. Curr Opin Neurol 1994;7:20-24.

20. Emiroglu MY, Bulut M, Sahin M, Acar G, Akcakoyun M, Kargin $\mathrm{R}$, et al. Assessment of atrial conduction time in patients with essential hypertension. J Electrocardiol 2011;44:251-256.

21. Ceylan O, Bayata S, Yeşil M, Arıkan E, Postacı N. Value of interatrial conduction time and $\mathrm{P}$ wave dispersion in the prediction of atrial fibrillation following coronary bypass surgery. Anadolu Kardiyol Derg 2010;10:495-501.

22. Cagirci G, Cay S, Gulsoy KG, Bayindir C, Vural MG, Firat H, et al. Tissue Doppler atrial conduction times and electrocardiogram interlead P-wave durations with varying severity of obstructive sleep apnea. J Electrocardiol 2011;44:478-482.

23. Emiroglu MY, Bulut M, Sahin M, Acar G, Akcakoyun M, Kargin $\mathrm{R}$, et al. Assessment of atrial conduction time in patients with essential hypertension. J Electrocardiol 2011;44:251-256.

24. Abhayaratna WP, Seward JB, Appleton CP, Douglas PS, Oh JK, Tajik AJ, et al. Left atrial size: physiologic determinants and clinical applications. J Am Coll Cardiol 2006;47:2357-2363.

25. Di Tullio MR, Jin Z, Russo C, Elkind MS, Rundek T, Yoshita $\mathrm{M}$, et al. Patent foramen ovale, subclinical cerebrovascular disease, and ischemic stroke in a population-based cohort. J Am Coll Cardiol 2013;62:35-41.

26. Lucas JF, Radtke WA, Bandisode VM, Fairbrother DL, Shirali GS. Characteristics of the inter-atrial communication in patients undergoing transcatheter device closure of atrial septal defects for cryptogenic stroke. Echocardiography 2005;22:814-817.

27. Goch A, Banach M, Piotrowski G, Szadkowska I, Goch JH. Echocardiographic evaluation of the left atrium and left atrial appendage function in patients with atrial septum aneurysm: implications for thromboembolic complications. Thorac Cardiovasc Surg 2007;55:365-370.

28. D’Andrea A, Caso P, Romano S, Scarafile R, Riegler L, Salerno $\mathrm{G}$, et al. Different effects of cardiac resynchronization therapy on left atrial function in patients with either idiopathic or ischaemic dilated cardiomyopathy: a two-dimensional speckle strain study. Eur Heart J 2007;28:2738-2748.

29. Hammerstingl C, Schwekendiek M, Momcilovic D, Schueler
R, Sinning JM, Schrickel JW, et al. Left atrial deformation imaging with ultrasound based two-dimensional speckle-tracking predicts the rate of recurrence of paroxysmal and persistent atrial fibrillation after successful ablation procedures. J Cardiovasc Electrophysiol 2012;23:247-255.

30. Debonnaire P, Thijssen J, Leong DP, Joyce E, Katsanos S, Hoogslag GE, et al. Global longitudinal strain and left atrial volume index improve prediction of appropriate implantable cardioverter defibrillator therapy in hypertrophic cardiomyopathy patients. Int J Cardiovasc Imaging 2014;30:549-558.

31. Antoni ML, ten Brinke EA, Atary JZ, Marsan NA, Holman ER, Schalij MJ, et al. Left atrial strain is related to adverse events in patients after acute myocardial infarction treated with primary percutaneous coronary intervention. Heart 2011;97:1332-1337.

32. Karabay CY, Zehir R, Güler A, Oduncu V, Kalayci A, Aung SM, et al. Left atrial deformation parameters predict left atrial appendage function and thrombus in patients in sinus rhythm with suspected cardioembolic stroke: a speckle tracking and transesophageal echocardiography study. Echocardiography 2013;30:572-581.

33. Pagola J, González-Alujas T, Flores A, Muchada M, RodriguezLuna D, Seró L, et al. Left atria strain is a surrogate marker for detection of atrial fibrillation in cryptogenic strokes. Stroke 2014; 45:e164-166.

34. Corradi D, Callegari S, Maestri R, Benussi S, Alfieri O. Structural remodeling in atrial fibrillation. Nat Clin Pract Cardiovasc Med 2008;5:782-796.

35. Gasparovic H, Cikes M, Kopjar T, Hlupic L, Velagic V, Milicic D, et al. Atrial apoptosis and fibrosis adversely affect atrial conduit, reservoir and contractile functions. Interact Cardiovasc Thorac Surg 2014;19:223-230.

36. Kono T, Morita H, Kuroiwa T, Onaka H, Takatsuka H, Fujiwara A. Left ventricular wall motion abnormalities in patients with subarachnoid hemorrhage: neurogenic stunned myocardium. J Am Coll Cardiol 1994;24:636-640.

37. James TN, Urthaler F, Isobe JH. Neurogenic influence on the atrial repolarization (P-Tp) segment. Am J Cardiol 1973;32: 799-807.

38. Chua HC, Sen S, Cosgriff RF, Gerstenblith G, Beauchamp NJ Jr, Oppenheimer SM. Neurogenic ST depression in stroke. Clin Neurol Neurosurg 1999;101:44-48. 